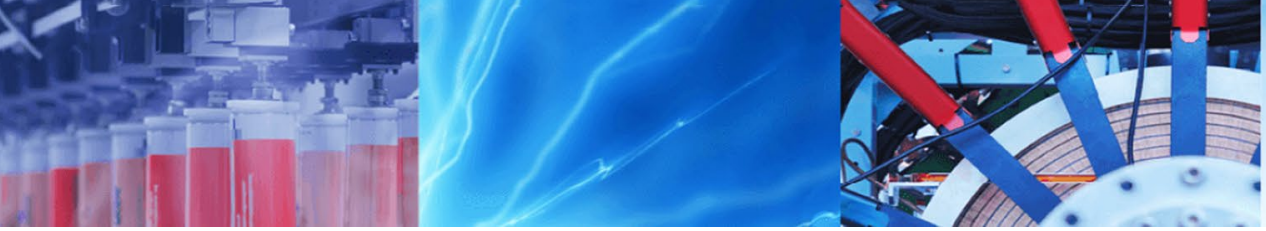

Research Article

\title{
Solving time-fractional Navier-Stokes equations using homotopy perturbation Elzaki transform
}

\author{
Rajarama Mohan Jena ${ }^{1} \cdot$ S. Chakraverty ${ }^{1}$
}

(c) Springer Nature Switzerland AG 2018

\begin{abstract}
In this article, a hybrid technique called homotopy perturbation Elzaki transform method has been applied to solve Navier-Stokes equation of fractional order. In the hybrid technique, homotopy perturbation method and Elzaki transform method are amalgamated. Three example problems are solved with a purpose to validate and demonstrate the efficacy of the present method. It is also demonstrated that the results obtained from the present method are in excellent agreement with the results by other methods. It is shown that the proposed method is found to be reliable, efficient and easy to implement for various related problems of science and engineering.
\end{abstract}

Keywords Elzaki transform - He's polynomials - Homotopy perturbation method - Caputo time-fractional derivative . Navier-Stokes equations

Mathematics Subject Classification 76D05 $\cdot 35$ N10

\section{Introduction}

Fractional calculus is an important branch of applied mathematics which deals with the differential and integral operators with non-integral powers. Fractional calculus has become popular due to its demonstrated wide range of application in rheology, viscoelasticity, electrochemistry, electromagnetism, fluid mechanics etc. For details, one may see the monographs of Kilbas et al. [1], some fundamental works on various aspects of fractional calculus are given by Kiryakova [2], Lakshmikantham and Vatsala [3], Miller and Ross [4] and the solutions method of differential equations of arbitrary real order and applications of the described methods in various fields are given by Podlubny [5]. In recent years, many analytical and approximate methods for solving fractional differential equations have been developed such as differential transform method [6, 7], finite difference method (FDM) [8], Adomian decomposition method (ADM) $[9,10]$, homotopy perturbation method (HPM) [11-13], Haar wavelet method (HWM) $[14,15]$, differential transform method (DTM) [16-18], variational iteration method (VIM) [19] and many others. Among all the above-listed methods, homotopy perturbation method which was first proposed by the Chinese researcher J.H. He in 1998 plays an important role. This is due to the fact that it addresses a problem directly without the need for any form of transformation, linearization and discrimination. Elzaki Transform (ET) is a new integral transform which was introduced by Tarig ELzaki in 2010. ET is modified transform of Sumudu and Laplace transforms. It is worth mentioning that there are some differential equations with variable coefficients which may not be solved by Sumudu and Laplace transforms but may easily be solved with the aid of ET. Fractional nonlinear differential equations have been solved by various authors [20] by means of the combination of ET and ADM. As regards, Klein-Gordon equations were solved by the authors [21] by amalgamation of ET and iterative method. Further,

S.Chakraverty, sne_chak@yahoo.com | Rajarama Mohan Jena, rajarama1994@gmail.com | ${ }^{1}$ Department of Mathematics, National Institute of Technology, Rourkela 769008, India.

SN Applied Sciences (2019) 1:16| https://doi.org/10.1007/s42452-018-0016-9

Received: 25 July 2018 / Accepted: 1 October 2018 / Published online: 17 October 2018

SN Applied Sciences

A SPRINGER NATURE journal 
non-linear partial differential equations are also solved by different authors $[22,23]$ using modified HPM.

The primary equation of movement of viscous fluid flow known as the NS equation has been presented in 1822 [24]. This equation portrays a few projections which include sea streams, fluid stream in channels, bloodstream and wind current around the wings of an airship. The NS equation was first carried out in 2005 in the fractional form in [25] by El-Shahed and Salem. The classical NS equation was answered by El-Shahed and Salem [25] by means of laplace transform (LT), finite Hankel transforms (FHT) and Fourier sine transform. A nonlinear fractional NS equation was solved analytically by Kumar et al. [26] by the combination of HPM with LT algorithm. Also the same NS equation was resolved by Ragab et al. [27] and Ganji et al. [28] by adopting homotopy analysis method. ADM was adopted by Birajdar [29] and Momani et al. [30] for the solution of fractional NS equation. Sunil Kumar et al. [31] achieved the analytical result of fractional NS equation by means of ADM and LT algorithm while Chaurasia and Kumar [32] solved the similar equation by the pairing of LT with FHT. The present paper gives an exact or approximate solution for the proposed problem by using HPETM.

This article is planned as follows: some basic features of fractional calculus related to the titled problems have been presented in Sect. 2. Elzaki transform and elaborated form of the HPETM have been included in Sects. 3 and 4 respectively. In Sect. 5, three example problems are included to validate the effectiveness and exactness of the proposed method. Lastly, a conclusion is given in Sect. 6.

\section{Basic features of fractional calculus}

Definition 2.1 The operator $D^{\alpha}$ of order $\alpha$ in Abel-Riemann $(A-R)$ sense is defined as $[4,5,40]$

$D^{\alpha} u(x)=\left\{\begin{array}{lc}\frac{d^{m}}{d x^{m}} u(x), & \alpha=m \\ \frac{1}{\Gamma(m-\alpha)} \frac{d}{d x^{m}} \int_{0}^{x} \frac{u(t)}{(x-t)^{\alpha-m+1}} d t, & m-1<\alpha<m\end{array}\right.$

where $m \in Z^{+}, \alpha \in R^{+}$and

$D^{-\alpha} u(x)=\frac{1}{\Gamma(\alpha)} \int_{0}^{x}(x-t)^{\alpha-1} u(t) d t, \quad 0<\alpha \leq 1$

Definition 2.2 The A-R fractional order integration operator $J^{\alpha}$ is described as $[4,5]$
$J^{\alpha} u(x)=\frac{1}{\Gamma(\alpha)} \int_{0}^{x}(x-t)^{\alpha-1} u(t) d t, t>0 \alpha>0$

Following Podlubny [5] we may have

$J^{\alpha} t^{n}=\frac{\Gamma(n+1)}{\Gamma(n+\alpha+1)} t^{n+\alpha}$

$D^{\alpha} t^{n}=\frac{\Gamma(n+1)}{\Gamma(n-\alpha+1)} t^{n-\alpha}$

Definition 2.3 The operator $D^{\alpha}$ of order $\alpha$ in Caputo sense is defined as $[5,33,39]$

${ }^{C} D^{\alpha} u(x)=\left\{\begin{array}{lc}\frac{1}{\Gamma(m-\alpha)} \int_{0}^{x} \frac{u^{m}(t)}{(x-t)^{\alpha-m+1}} d t, & m-1<\alpha<m, \\ \frac{d^{m}}{d t^{m}} u(x), & \alpha=m\end{array}\right.$

Definition 2.4 [4, 5, 33, 34]:

(a) $D_{t}^{\alpha} J_{t}^{\alpha} f(t)=f(t)$

(b) $J_{t}^{\alpha} D_{t}^{\alpha} f(t)=f(t)-\sum_{k=0}^{m} f^{(k)}\left(0^{+}\right) \frac{t^{k}}{k !}$, for $t>0$, and $m-1<\alpha \leq m, m \in N$.

\section{Elzaki transform (ET)}

The definition of modified Sumudu transform or ET of the function $f(t)$ is defined as

$E[f(t)]=F(q)=q \int_{0}^{\infty} f(t) e^{\frac{-t}{q}} d t, t>0$

The Elzaki transform is very effective and powerful method for solving integral equation which cannot be solved by the Sumudu transform method. For this, one may see the Ref. [35].

Integration by parts in Eq. (8) can be used in order to find ET of partial derivatives as follows [35].

1. $E\left[\frac{\partial f(x, t)}{\partial t}\right]=\frac{1}{q} F(x, q)-q f(x, 0)$

2. $E\left[\frac{\partial^{2} f(x, t)}{\partial t^{2}}\right]=\frac{1}{q^{2}} F(x, q)-f(x, 0)-q \frac{\partial f(x, 0)}{\partial t}$

3. $E\left[\frac{\partial f(x, t)}{\partial x}\right]=\frac{d}{d x} F(x, q)$ 
4. $E\left[\frac{\partial^{2} f(x, t)}{\partial x^{2}}\right]=\frac{d^{2}}{d x^{2}} F(x, q)$.

\subsection{ET of Caputo fractional derivative}

Theorem 1 ([36]) If $G(s)$ is the Laplace transform off $(t)$ then $\operatorname{ETF}(q)$ of $f(t)$ is given by

$F(q)=q G\left(\frac{1}{q}\right)$

Theorem 2 ([36]) If $F(q)$ is the ET of the function $f(t)$ then

$$
E\left[D^{\alpha} f(t)\right]=\frac{F(q)}{q^{\alpha}}-\sum_{k=0}^{n-1} q^{k-\alpha+2} f^{(k)}(0), n-1<\alpha \leq n
$$

\section{Homotopy perturbation Elzaki transform method (HPETM)}

In order to clarify the idea of HPETM, the fractional order nonlinear non-homogeneous partial differential equation with initial condition (IC) is consider as below

$D_{t}^{\alpha} f(x, y, z, t)+R f(x, y, z, t)+N f(x, y, z, t)=g(x, y, z, t), 0<\alpha \leq 1$

$f(x, y, z, 0)=h(x, y, z)$

where $D_{t}^{\alpha} f(x, y, z, t)$ is the derivative of $f(x, y, z, t)$ in Caputo sense, $R, N$ are the linear and nonlinear differential operators and $g(x, y, z, t)$ is the source term.

Now by taking ET on both sides of Eq. (11), we have

$E\left[D_{t}^{\alpha} f(x, y, z, t)+R f(x, y, z, t)+N f(x, y, z, t)\right]=E[g(x, y, z, t)]$

Using differentiation property of ET, we obtain

$$
\begin{aligned}
E\{f(x, y, z, t)\}= & \sum_{k=0}^{n-1} q^{k+2} f^{(k)}(0)+q^{\alpha}[E\{g(x, y, z, t)\} \\
& -E\{R f(x, y, z, t)+N f(x, y, z, t)\}]
\end{aligned}
$$

Applying inverse Elzaki transform on both sides of Eqs. (14) and (12), we find

$f(x, y, z, t)=G(x, y, z, t)-E^{-1}\left[q^{\alpha}[E\{R f(x, y, z, t)+N f(x, y, z, t)\}]\right]$

where $G(x, y, z, t)$ represents the term coming from initial condition and source term.

Now, by applying HPM to the Eq. (15), we get

$$
\begin{aligned}
& f(x, y, z, t)=G(x, y, z, t) \\
& \quad-p\left(E^{-1}\left[q^{\alpha}[E\{R f(x, y, z, t)+N f(x, y, z, t)\}]\right]\right) .
\end{aligned}
$$

The homotopy parameter $p$ is used to expand the solution as

$f(x, y, z, t)=\sum_{n=0}^{\infty} p^{n} f_{n}(x, y, z, t)$

and the nonlinear term is decomposed as

$N f(x, y, z, t)=\sum_{n=0}^{\infty} p^{n} H_{n}(f)$

where $H_{n}(f)$ is He's polynomials and is given by

$H_{n}\left(f_{0}, f_{1}, \ldots, f_{n}\right)=\frac{1}{n !} \frac{\partial}{\partial p^{n}}\left[N\left(\sum_{n=0}^{\infty} p^{n} f_{n}\right)\right]$.

Substituting Eqs. (17) and (18) in Eq. (16), we get

$$
\begin{aligned}
& \sum_{n=0}^{\infty} p^{n} f_{n}(x, y, z, t)=G(x, y, z, t) \\
& -p\left(E^{-1}\left[q^{\alpha}\left[E\left\{R \sum_{n=0}^{\infty} p^{n} f_{n}(x, y, z, t)+N \sum_{n=0}^{\infty} p^{n} H_{n}(f)\right\}\right]\right]\right)
\end{aligned}
$$

Comparing the coefficient of equal powers of $p$ from both sides of above equation, the following equations are obtained

$p^{0}: f_{0}(x, y, z, t)=G(x, y, z, t)$,

$p^{1}: f_{1}(x, y, z, t)=E^{-1}\left[q^{\alpha}\left[E\left\{R f_{0}(x, y, z, t)+H_{0}(f)\right\}\right]\right]$

$p^{2}: f_{2}(x, y, z, t)=E^{-1}\left[q^{\alpha}\left[E\left\{R f_{1}(x, y, z, t)+H_{1}(f)\right\}\right]\right]$

Continuing in this manner we may find $f_{n}(x, y, z, t)$ and then the solution is written as

$f(x, y, z, t)=f_{1}(x, y, z, t)+f_{2}(x, y, z, t)+f_{3}(x, y, z, t)+\cdots$

\section{Application of HPETM on NS equation}

The proposed method is implemented here and then the accuracy of the HPETM is investigated for NS equation. The time fractional NS equation with constant density $\rho$ and kinematic viscosity $v=\frac{\eta}{\rho}$ is given as $[24,29]$

$\left\{\begin{array}{lr}D_{t}^{\alpha} U+(U . \nabla) U=\rho_{0} \nabla^{2} U-\frac{1}{\rho} \nabla \rho, & \Omega \times(0, T) \\ \nabla . U=0, & \Omega \times(0, T) \\ U=0, & \Omega \times(0, T)\end{array}\right.$ 
where $U=(u, v, w), t, \rho$ represent the fluid vector, time and pressure respectively. $\eta$ is the dynamic viscosity while the ratio $\rho_{0}=\frac{\eta}{\rho}$ represents the kinematics viscosity. Here $\Omega=(-\pi, \pi) \times(-\pi, \pi)$ is the domain with boundary $\partial \Omega$. In Cartesian coordinate, Eq. (21) is written as

$\left\{\begin{array}{l}D_{t}^{\alpha} u+u \frac{\partial u}{\partial x}+v \frac{\partial u}{\partial y}+w \frac{\partial u}{\partial z}=\rho_{0}\left(\frac{\partial^{2} u}{\partial x^{2}}+\frac{\partial^{2} u}{\partial y^{2}}+\frac{\partial^{2} u}{\partial z^{2}}\right)-\frac{1}{\rho} \frac{\partial p}{\partial x}, \\ D_{t}^{\alpha} v+u \frac{\partial v}{\partial x}+v \frac{\partial v}{\partial y}+w \frac{\partial v}{\partial z}=\rho_{0}\left(\frac{\partial^{2} v}{\partial x^{2}}+\frac{\partial^{2} v}{\partial y^{2}}+\frac{\partial^{2} v}{\partial z^{2}}\right)-\frac{1}{\rho} \frac{\partial p}{\partial y}, \\ D_{t}^{\alpha} w+u \frac{\partial w}{\partial x}+v \frac{\partial w}{\partial y}+w \frac{\partial w}{\partial z}=\rho_{0}\left(\frac{\partial^{2} w}{\partial x^{2}}+\frac{\partial^{2} w}{\partial y^{2}}+\frac{\partial^{2} w}{\partial z^{2}}\right)-\frac{1}{\rho} \frac{\partial p}{\partial z},\end{array}\right.$

If the value of $p$ is known then all the values of $g_{1}=-\frac{1}{\rho} \frac{\partial p}{\partial x}, g_{2}=-\frac{1}{\rho} \frac{\partial p}{\partial y} \quad$ and $\quad g_{3}=-\frac{1}{\rho} \frac{\partial p}{\partial z} \quad$ can be determined.

\subsection{Numerical examples}

Example 1 From Eq. (22), 2-dimensional NS equation of fractional order with $g_{1}=-g_{2}=g$ may be written as

$D_{t}^{\alpha} u+u \frac{\partial u}{\partial x}+v \frac{\partial u}{\partial y}=\rho_{0}\left(\frac{\partial^{2} u}{\partial x^{2}}+\frac{\partial^{2} u}{\partial y^{2}}\right)+g$

$D_{t}^{\alpha} v+u \frac{\partial v}{\partial x}+v \frac{\partial v}{\partial y}=\rho_{0}\left(\frac{\partial^{2} v}{\partial x^{2}}+\frac{\partial^{2} v}{\partial y^{2}}\right)-g$

with IC [37]

$u(x, y, 0)=-\sin (x+y), v(x, y, 0)=\sin (x+y)$.

Applying ET on both sides of Eq. (23) with IC (24), we get

$$
\begin{aligned}
E\{u(x, y, t)\}= & -q^{2} \sin (x+y) \\
& +q^{\alpha} E\left[\rho_{0}\left(\frac{\partial^{2} u}{\partial x^{2}}+\frac{\partial^{2} u}{\partial y^{2}}\right)+g-u \frac{\partial u}{\partial x}-v \frac{\partial u}{\partial y}\right]
\end{aligned}
$$

$$
\begin{aligned}
E\{v(x, y, t)\}= & q^{2} \sin (x+y) \\
& +q^{\alpha} E\left[\rho_{0}\left(\frac{\partial^{2} v}{\partial x^{2}}+\frac{\partial^{2} v}{\partial y^{2}}\right)-g-u \frac{\partial v}{\partial x}-v \frac{\partial v}{\partial y}\right]
\end{aligned}
$$

The inverse Elzaki transform of Eqs. (25) and (26) implies that

$$
\begin{aligned}
& u(x, y, t)=-\sin (x+y) \\
& +E^{-1}\left[q^{\alpha} E\left[\rho_{0}\left(\frac{\partial^{2} u}{\partial x^{2}}+\frac{\partial^{2} u}{\partial y^{2}}\right)+g-u \frac{\partial u}{\partial x}-v \frac{\partial u}{\partial y}\right]\right]
\end{aligned}
$$

$$
\begin{aligned}
& v(x, y, t)=\sin (x+y) \\
& \quad+E^{-1}\left[q^{\alpha} E\left[\rho_{0}\left(\frac{\partial^{2} v}{\partial x^{2}}+\frac{\partial^{2} v}{\partial y^{2}}\right)-g-u \frac{\partial v}{\partial x}-v \frac{\partial v}{\partial y}\right]\right]
\end{aligned}
$$

Simplifying Eqs. (27) and (28), we get

$$
\begin{aligned}
& u(x, y, t)=-\sin (x+y)+\frac{g t^{\alpha}}{\Gamma(\alpha+1)} \\
& +E^{-1}\left[q^{\alpha} E\left[\rho_{0}\left(\frac{\partial^{2} u}{\partial x^{2}}+\frac{\partial^{2} u}{\partial y^{2}}\right)-u \frac{\partial u}{\partial x}-v \frac{\partial u}{\partial y}\right]\right] \\
& v(x, y, t)=\sin (x+y)-\frac{g t^{\alpha}}{\Gamma(\alpha+1)} \\
& +E^{-1}\left[q^{\alpha} E\left[\rho_{0}\left(\frac{\partial^{2} v}{\partial x^{2}}+\frac{\partial^{2} v}{\partial y^{2}}\right)-u \frac{\partial v}{\partial x}-v \frac{\partial v}{\partial y}\right]\right]
\end{aligned}
$$

Now applying the homotopy perturbation method, we have

$$
\begin{aligned}
\sum_{n=0}^{\infty} p^{n} u_{n}(x, y, t)= & -\sin (x+y)+\frac{g t^{\alpha}}{\Gamma(\alpha+1)} \\
& +p\left\{E^{-1}\left[q^{\alpha} E\left[\sum_{n=0}^{\infty} p^{n} H_{n}(u)\right]\right]\right\} \\
\sum_{n=0}^{\infty} p^{n} v_{n}(x, y, t)= & \sin (x+y)-\frac{g t^{\alpha}}{\Gamma(\alpha+1)} \\
& +p\left\{E^{-1}\left[q^{\alpha} E\left[\sum_{n=0}^{\infty} p^{n} H_{n}(v)\right]\right]\right\}
\end{aligned}
$$

where $H_{n}(u)$ and $H_{n}(v)$ are He's polynomials which signifies the nonlinear terms.

$$
\left\{\begin{array}{l}
H_{n}(u)=p\left[\rho_{0}\left(\frac{\partial^{2} u}{\partial x^{2}}+\frac{\partial^{2} u}{\partial y^{2}}\right)-u \frac{\partial u}{\partial x}-v \frac{\partial u}{\partial y}\right]=0 \\
H_{n}(v)=p\left[\rho_{0}\left(\frac{\partial^{2} v}{\partial x^{2}}+\frac{\partial^{2} v}{\partial y^{2}}\right)-u \frac{\partial v}{\partial x}-v \frac{\partial v}{\partial y}\right]=0
\end{array}\right.
$$

where

$$
\begin{aligned}
& u=u_{0}+p u_{1}+p^{2} u_{2}+\cdots \\
& v=v_{0}+p v_{1}+p^{2} v_{2}+\cdots
\end{aligned}
$$

The first few components of He's polynomials are given as

$$
\left\{\begin{array}{c}
H_{0}(u)=\rho_{0}\left(u_{0 x x}+u_{0 y y}\right)-u_{0} u_{0 x}-v_{0} u_{0 y} \\
H_{0}(v)=\rho_{0}\left(v_{0 x x}+v_{0 y y}\right)-u_{0} v_{0 x}-v_{0} v_{0 y} \\
H_{1}(u)=\rho_{0}\left(u_{1 x x}+u_{1 y y}\right)-u_{0} u_{1 x}-u_{1} u_{0 x}-v_{0} u_{1 y}-v_{1} u_{0 y} \\
H_{1}(v)=\rho_{0}\left(v_{1 x x}+v_{1 y y}\right)-u_{0} v_{1 x}-u_{1} v_{0 x}-v_{0} v_{1 y}-v_{1} v_{0 y} \\
\vdots
\end{array}\right.
$$


Using the above He's polynomials and comparing the coefficients of same power of $p$ in Eqs. (31) and (32) we have

$$
\begin{gathered}
p^{0}: u_{0}(x, y, t)=-\sin (x+y)+\frac{g t^{\alpha}}{\Gamma(\alpha+1)} \\
v_{0}(x, y, t)=\sin (x+y)-\frac{g t^{\alpha}}{\Gamma(\alpha+1)}
\end{gathered}
$$

$p^{1}: u_{1}(x, y, t)=E^{-1}\left[q^{\alpha} E\left[H_{0}(u)\right]\right]=2 \rho_{0} \sin (x+y) \frac{t^{\alpha}}{\Gamma(\alpha+1)}$

$$
v_{1}(x, y, t)=E^{-1}\left[q^{\alpha} E\left[H_{0}(v)\right]\right]=-2 \rho_{0} \sin (x+y) \frac{t^{\alpha}}{\Gamma(\alpha+1)}
$$

$$
\begin{aligned}
p^{2}: u_{2}(x, y, t) & =E^{-1}\left[q^{\alpha} E\left[H_{1}(u)\right]\right]=-4 \rho_{0}^{2} \sin (x+y) \frac{t^{2 \alpha}}{\Gamma(2 \alpha+1)} \\
v_{2}(x, y, t) & =E^{-1}\left[q^{\alpha} E\left[H_{1}(v)\right]\right]=4 \rho_{0}^{2} \sin (x+y) \frac{t^{2 \alpha}}{\Gamma(2 \alpha+1)}
\end{aligned}
$$

So the solution $u(x, y, t)$ and $v(x, y, t)$ are written as

$$
\begin{aligned}
u(x, y, t)= & u_{0}(x, y, t)+u_{1}(x, y, t)+u_{2}(x, y, t)+\cdots \\
& =-\sin (x+y) e^{-2 \rho_{0} t^{\alpha}}+\frac{g t^{\alpha}}{\Gamma(\alpha+1)} \\
v(x, y, t)= & v_{0}(x, y, t)+v_{1}(x, y, t)+v_{2}(x, y, t)+\cdots \\
= & \sin (x+y) e^{-2 \rho_{0} t^{\alpha}}-\frac{g t^{\alpha}}{\Gamma(\alpha+1)}
\end{aligned}
$$

For $g=0, \alpha=1$ Eqs. (33) and (34) reduce to

$\left\{\begin{array}{l}u(x, y, t)=-\sin (x+y) e^{-2 \rho_{0} t} \\ v(x, y, t)=\sin (x+y) e^{-2 \rho_{0} t}\end{array}\right.$

This solution is in good agreement with [37]. The plots of Eq. (35) are depicted in Figs. 1, 2, 3 and 4, for different values of $\alpha=1,0.2,0.4,0.6, \rho_{0}=0.5, t=3$. The comparison plots of $U_{0}, U_{1}, U_{2}, U_{3}, U_{4}$ and $V_{0}, V_{1}, V_{2}, V_{3}, V_{4}$ with their exact solution (35) for $\alpha=1$ are depicted in Fig. 5 and solution plots of Example 1 are given in Fig. 6 for different values of $\alpha$.

Example 2 Consider the NS Eq. (23) with IC [37]

$u(x, y, 0)=-e^{x+y}, v(x, y, 0)=e^{x+y}$

Applying ET on both sides of Eq. (23) subject to IC (36), we get

$E\{u(x, y, t)\}=-q^{2} e^{x+y}+q^{\alpha} E\left[\rho_{0}\left(\frac{\partial^{2} u}{\partial x^{2}}+\frac{\partial^{2} u}{\partial y^{2}}\right)+g-u \frac{\partial u}{\partial x}-v \frac{\partial u}{\partial y}\right]$

$E\{v(x, y, t)\}=q^{2} e^{x+y}+q^{\alpha} E\left[\rho_{0}\left(\frac{\partial^{2} v}{\partial x^{2}}+\frac{\partial^{2} v}{\partial y^{2}}\right)-g-u \frac{\partial v}{\partial x}-v \frac{\partial v}{\partial y}\right]$

The inverse Elzaki transform of Eqs. (37) and (38) implies that
Fig. 1 Solution plots of Eq. (35) for $\rho_{0}=0.5, t=3, \alpha=1$ and $g=0$

Fig. 2 Solution plots of Eq. (35) for $\rho_{0}=0.5, t=3, \alpha=0.2$ and $g=0$ (Example 1)
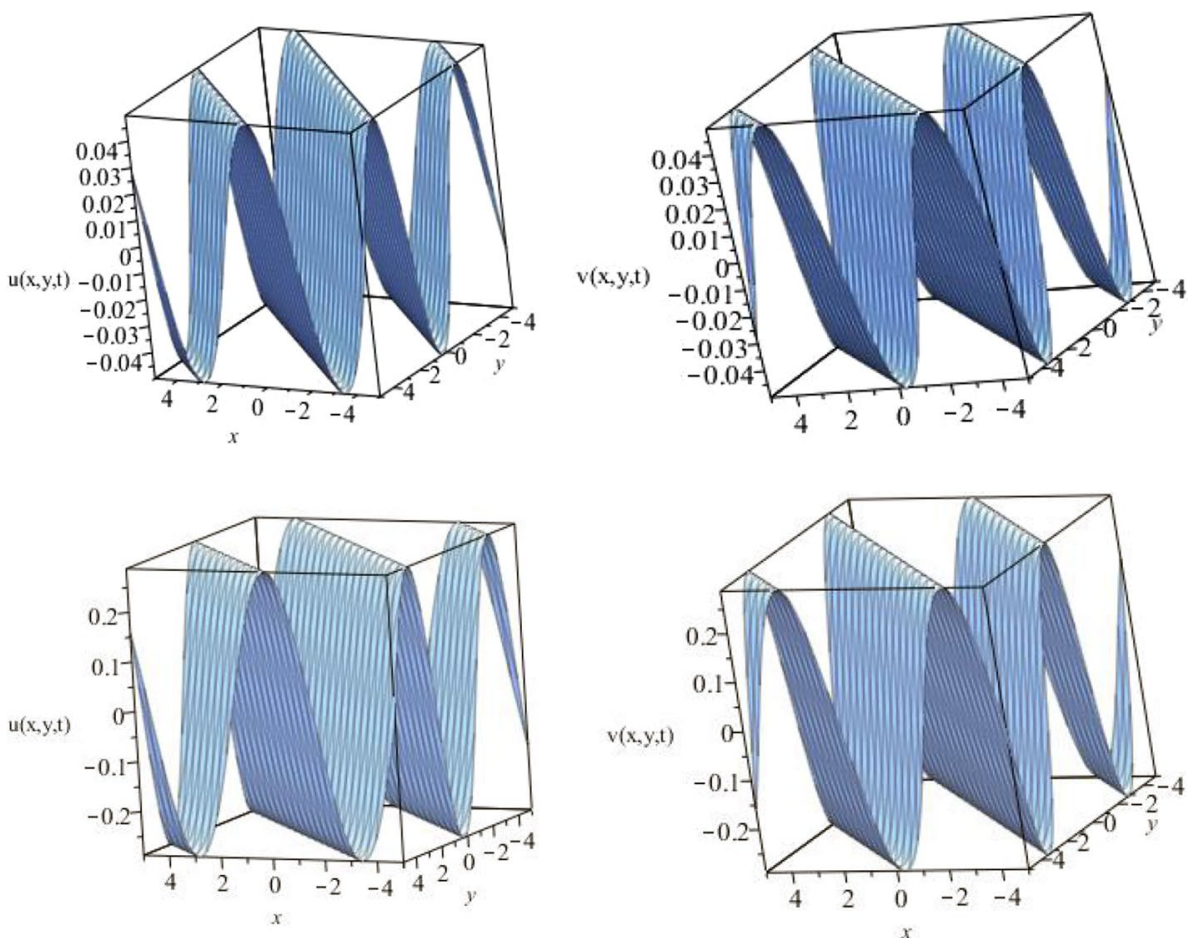

SN Applied Sciences A SPRINGER NATURE journal 
Fig. 3 Solution plots of Eq. (35) for $\rho_{0}=0.5, t=3, \alpha=0.4$ and $g=0$ (Example 1)

Fig. 4 Solution plots of Eq. (35) for $\rho_{0}=0.5, t=3, \alpha=0.6$ and $g=0$ (Example 1)
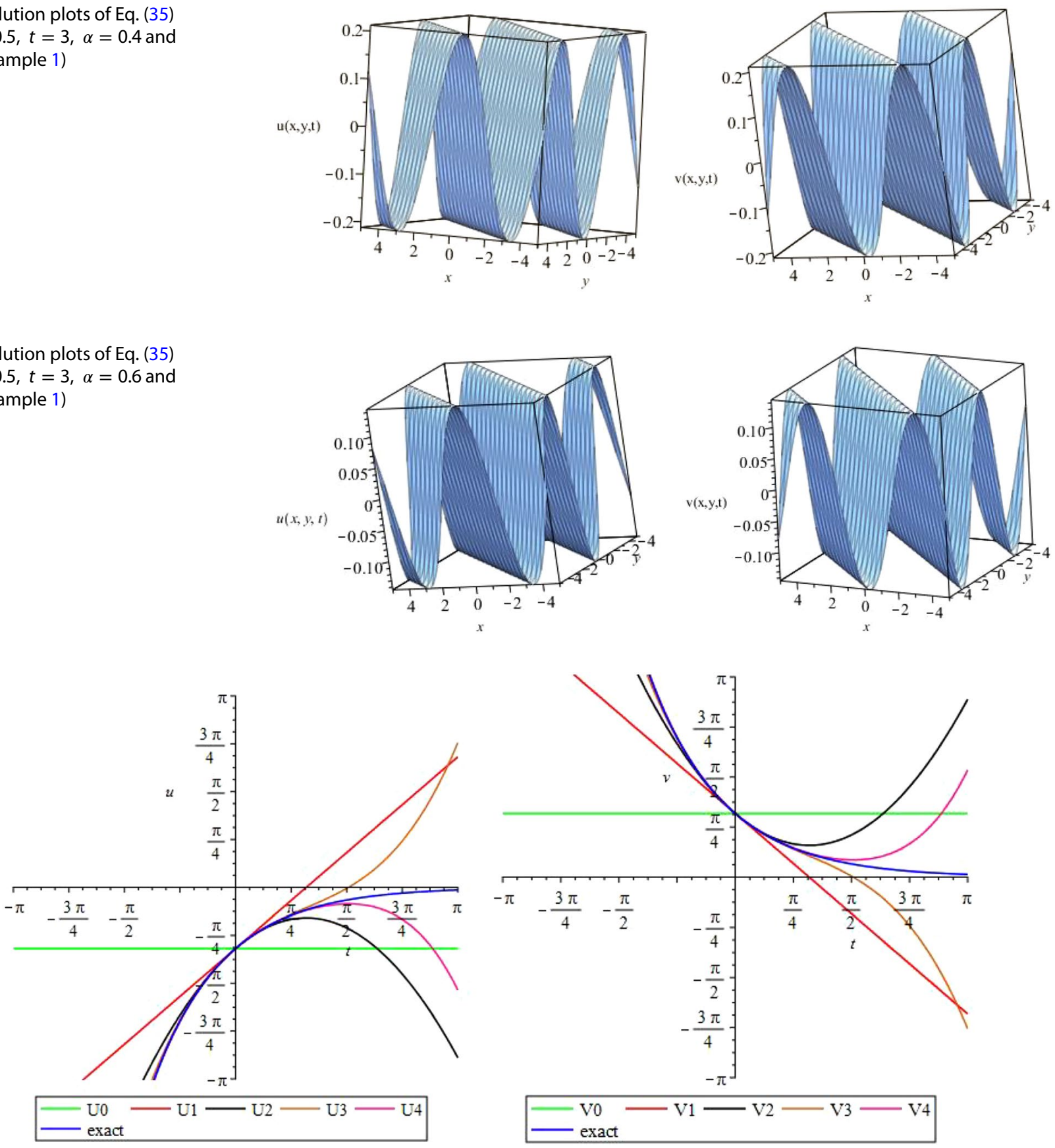

Fig. 5 Comparison of the approximate solution $U_{0}, U_{1}, U_{2}, U_{3}, U_{4}$ and $V_{0}, V_{1}, V_{2}, V_{3}, V_{4}$ with their exact solution (35) for $\rho_{0}=0.5, x=\frac{\pi}{4}, y=\frac{\pi}{4}$ (Example 1)

$u(x, y, t)=-e^{x+y}+E^{-1}\left[q^{\alpha} E\left[\rho_{0}\left(\frac{\partial^{2} u}{\partial x^{2}}+\frac{\partial^{2} u}{\partial y^{2}}\right)+g-u \frac{\partial u}{\partial x}-v \frac{\partial u}{\partial y}\right]\right]$

$v(x, y, t)=e^{x+y}+E^{-1}\left[q^{\alpha} E\left[\rho_{0}\left(\frac{\partial^{2} v}{\partial x^{2}}+\frac{\partial^{2} v}{\partial y^{2}}\right)-g-u \frac{\partial v}{\partial x}-v \frac{\partial v}{\partial y}\right]\right]$
Simplifying Eqs. (39) and (40), we get

$$
\begin{aligned}
& u(x, y, t)=-e^{x+y}+\frac{g t^{\alpha}}{\Gamma(\alpha+1)} \\
& +E^{-1}\left[q^{\alpha} E\left[\rho_{0}\left(\frac{\partial^{2} u}{\partial x^{2}}+\frac{\partial^{2} u}{\partial y^{2}}\right)-u \frac{\partial u}{\partial x}-v \frac{\partial u}{\partial y}\right]\right]
\end{aligned}
$$



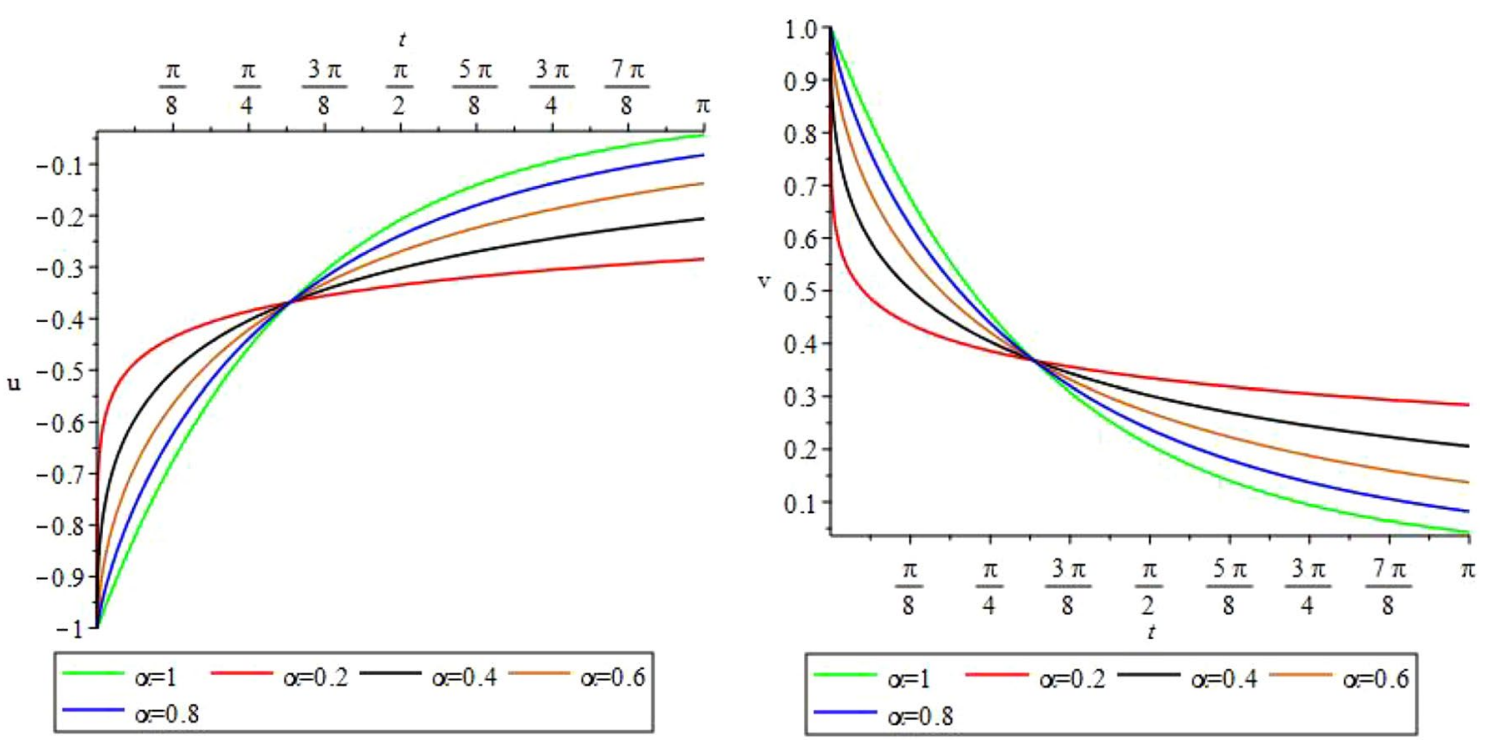

Fig. 6 Solution plots of Eq. (35) for different values of $\alpha$ (Example 1)

$$
\begin{aligned}
& v(x, y, t)=e^{x+y}-\frac{g t^{\alpha}}{\Gamma(\alpha+1)} \\
& +E^{-1}\left[q^{\alpha} E\left[\rho_{0}\left(\frac{\partial^{2} v}{\partial x^{2}}+\frac{\partial^{2} v}{\partial y^{2}}\right)-u \frac{\partial v}{\partial x}-v \frac{\partial v}{\partial y}\right]\right]
\end{aligned}
$$

Now applying HPM, we have

$$
\begin{aligned}
& \sum_{n=0}^{\infty} p^{n} u_{n}(x, y, t)=-e^{x+y}+\frac{g t^{\alpha}}{\Gamma(\alpha+1)} \\
& +p\left\{E^{-1}\left[q^{\alpha} E\left[\sum_{n=0}^{\infty} p^{n} H_{n}(u)\right]\right]\right\} \\
& \sum_{n=0}^{\infty} p^{n} v_{n}(x, y, t)=e^{x+y}-\frac{g t^{\alpha}}{\Gamma(\alpha+1)}+p\left\{E^{-1}\left[q^{\alpha} E\left[\sum_{n=0}^{\infty} p^{n} H_{n}(v)\right]\right]\right\}
\end{aligned}
$$

where $H_{n}(u)$ and $H_{n}(v)$ are He's polynomials that denotes the nonlinear terms and are given as

$$
\left\{\begin{array}{l}
H_{n}(u)=p\left[\rho_{0}\left(\frac{\partial^{2} u}{\partial x^{2}}+\frac{\partial^{2} u}{\partial y^{2}}\right)-u \frac{\partial u}{\partial x}-v \frac{\partial u}{\partial y}\right]=0 \\
H_{n}(v)=p\left[\rho_{0}\left(\frac{\partial^{2} v}{\partial x^{2}}+\frac{\partial^{2} v}{\partial y^{2}}\right)-u \frac{\partial v}{\partial x}-v \frac{\partial v}{\partial y}\right]=0
\end{array}\right.
$$

where

$$
\begin{aligned}
& u=u_{0}+p u_{1}+p^{2} u_{2}+\cdots \\
& v=v_{0}+p v_{1}+p^{2} v_{2}+\cdots
\end{aligned}
$$

The first few components of He's polynomials are given by

$$
\left\{\begin{array}{c}
H_{0}(u)=\rho_{0}\left(u_{0 x x}+u_{0 y y}\right)-u_{0} u_{0 x}-v_{0} u_{0 y} \\
H_{0}(v)=\rho_{0}\left(v_{0 x x}+v_{0 y y}\right)-u_{0} v_{0 x}-v_{0} v_{0 y} \\
H_{1}(u)=\rho_{0}\left(u_{1 x x}+u_{1 y y}\right)-u_{0} u_{1 x}-u_{1} u_{0 x}-v_{0} u_{1 y}-v_{1} u_{0 y} \\
H_{1}(v)=\rho_{0}\left(v_{1 x x}+v_{1 y y}\right)-u_{0} v_{1 x}-u_{1} v_{0 x}-v_{0} v_{1 y}-v_{1} v_{0 y} \\
\vdots
\end{array}\right.
$$

Using the above He's polynomials and comparing the coefficients of same power of $p$ in Eqs. (43) and (44) we have

$$
\begin{aligned}
p^{0}: u_{0}(x, y, t) & =-e^{x+y}+\frac{g t^{\alpha}}{\Gamma(\alpha+1)} \\
v_{0}(x, y, t) & =e^{x+y}-\frac{g t^{\alpha}}{\Gamma(\alpha+1)} \\
p^{1}: u_{1}(x, y, t) & =E^{-1}\left[q^{\alpha} E\left[H_{0}(u)\right]\right]=-2 \rho_{0} e^{x+y} \frac{t^{\alpha}}{\Gamma(\alpha+1)} \\
v_{1}(x, y, t) & =E^{-1}\left[q^{\alpha} E\left[H_{0}(v)\right]\right]=2 \rho_{0} e^{x+y} \frac{t^{\alpha}}{\Gamma(\alpha+1)} \\
p^{2}: u_{2}(x, y, t) & =E^{-1}\left[q^{\alpha} E\left[H_{1}(u)\right]\right]=-4 \rho_{0}^{2} e^{x+y} \frac{t^{2 \alpha}}{\Gamma(2 \alpha+1)} \\
v_{2}(x, y, t) & =E^{-1}\left[q^{\alpha} E\left[H_{1}(v)\right]\right]=4 \rho_{0}^{2} e^{x+y} \frac{t^{2 \alpha}}{\Gamma(2 \alpha+1)}
\end{aligned}
$$

Then the solution $u(x, y, t)$ and $v(x, y, t)$ are given as 


$$
\begin{aligned}
u(x, y, t) & =u_{0}(x, y, t)+u_{1}(x, y, t)+u_{2}(x, y, t)+\cdots \\
& =-e^{x+y} e^{2 \rho_{0} t^{\alpha}}+\frac{g t^{\alpha}}{\Gamma(\alpha+1)} \\
v(x, y, t) & =v_{0}(x, y, t)+v_{1}(x, y, t)+v_{2}(x, y, t)+\cdots \\
& =e^{x+y} e^{2 \rho_{0} t^{\alpha}}-\frac{g t^{\alpha}}{\Gamma(\alpha+1)}
\end{aligned}
$$

For $g=0, \alpha=1$ Eqs. (45) and (46) reduce to

$$
\left\{\begin{array}{l}
u(x, y, t)=-e^{x+y+2 \rho_{0} t} \\
v(x, y, t)=e^{x+y+2 \rho_{0} t}
\end{array}\right.
$$

This solution is same as the solution solved in [29]. The behavior of the solution (47) are depicted in Figs. 7, 8 and 9 for different values of $\alpha=1,0.4,0.8, \rho_{0}=0.5, t=0.05$. The comparison plots of $U_{0}, U_{1}, U_{2}, U_{3}, U_{4}$ and $V_{0}, V_{1}, V_{2}, V_{3}, V_{4}$

with their exact solution (47) for $\alpha=1$ are depicted in Fig. 10 and solution of plots of Example 2 for different values of $\alpha$ are illustrated in Fig. 11

Example 3 Finally let us consider 3-dimensional NS Eq. (22) with $g_{1}=g_{2}=g_{3}=0$ with IC [37]

$$
\begin{aligned}
u(x, y, z, 0) & =-0.5 x+y+z, v(x, y, z, 0) \\
& =x-0.5 y+z, w(x, y, z, 0) \\
& =x+y-0.5 z
\end{aligned}
$$

Applying ET on both sides of Eq. (22) subject to IC (48), we have

$$
\begin{aligned}
& E\{u(x, y, z, t)\}=q^{2}(-0.5 x+y+z) \\
& \quad+q^{\alpha} E\left[\rho_{0}\left(\frac{\partial^{2} u}{\partial x^{2}}+\frac{\partial^{2} u}{\partial y^{2}}+\frac{\partial^{2} u}{\partial z^{2}}\right)-u \frac{\partial u}{\partial x}-v \frac{\partial u}{\partial y}-w \frac{\partial u}{\partial z}\right]
\end{aligned}
$$

Fig. 7 Solution plots of Eq. (47) for $\rho_{0}=0.5, t=0.05, \alpha=1$ and $g=0$ (Example 2)

Fig. 8 Solution plots of Eq. (47) for $\rho_{0}=0.5, t=0.05, \alpha=0.4$ and $g=0$ (Example 2)

Fig. 9 Solution plots of Eq. (47) for $\rho_{0}=0.5, t=0.05, \alpha=0.8$ and $g=0$ (Example 2)
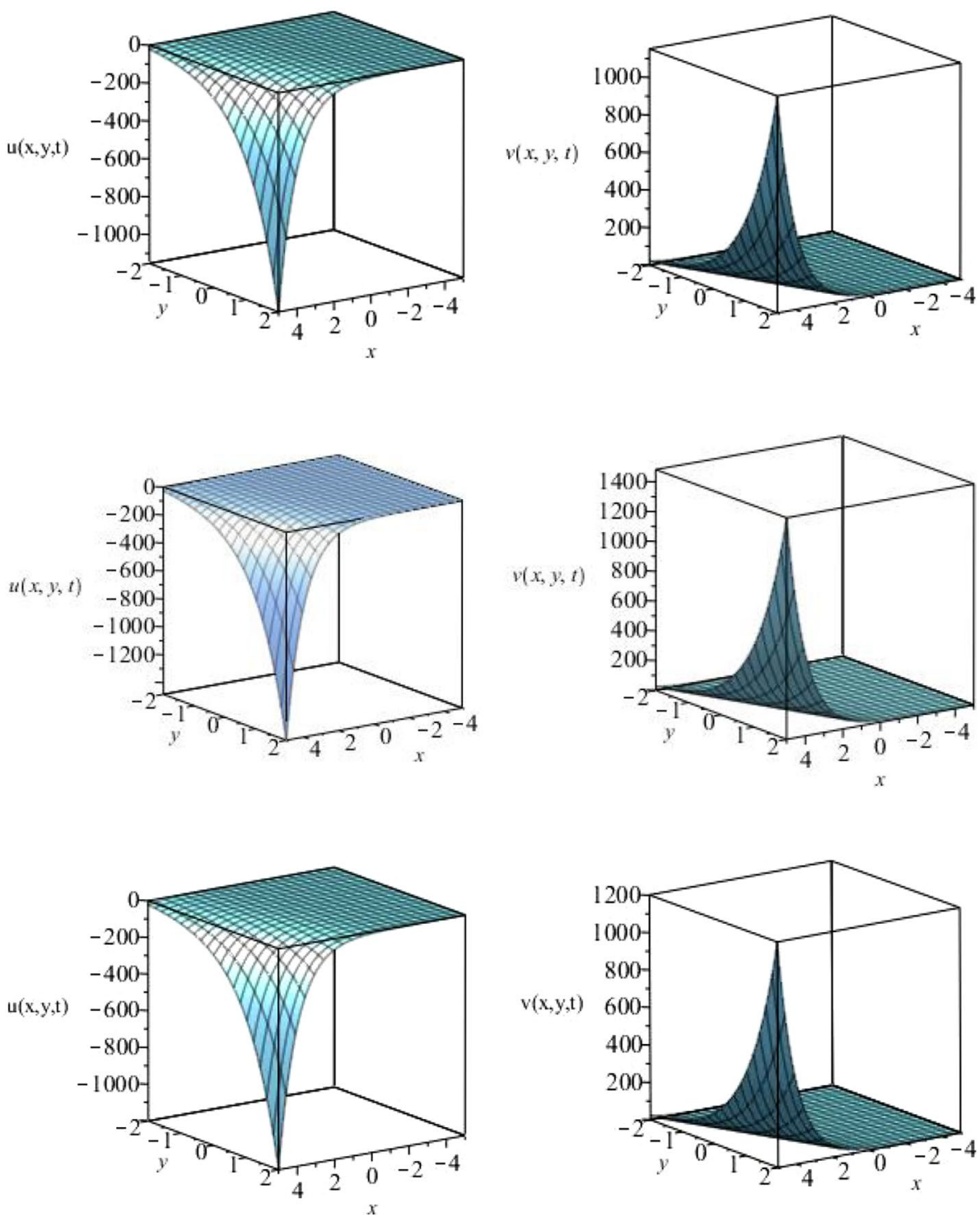

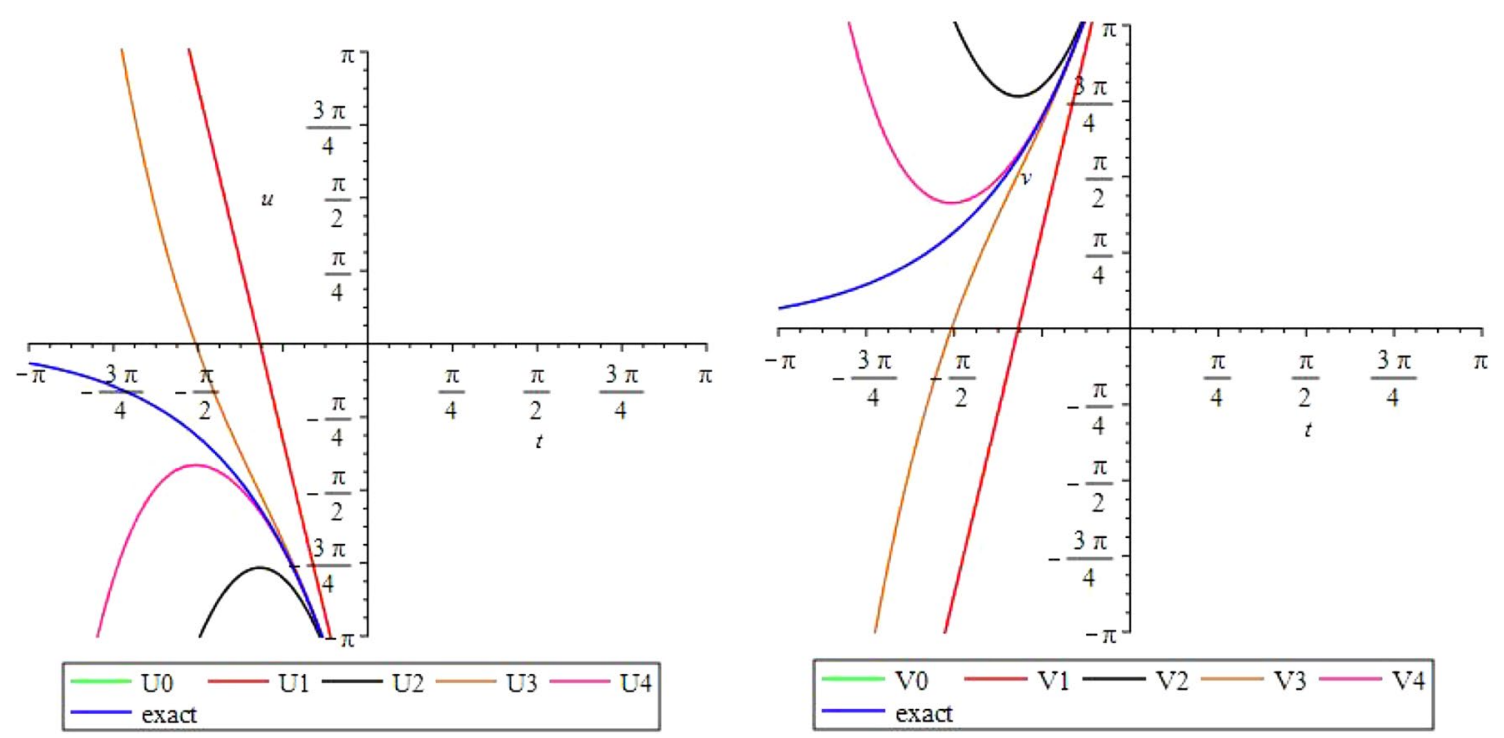

Fig. 10 Comparison of the approximate solution $U_{0}, U_{1}, U_{2}, U_{3}, U_{4}$ and $V_{0}, V_{1}, V_{2}, V_{3}, V_{4}$ with their exact solution (47) for $\rho_{0}=0.5, x=\frac{\pi}{4}, y=\frac{\pi}{4}$ (Example 2)
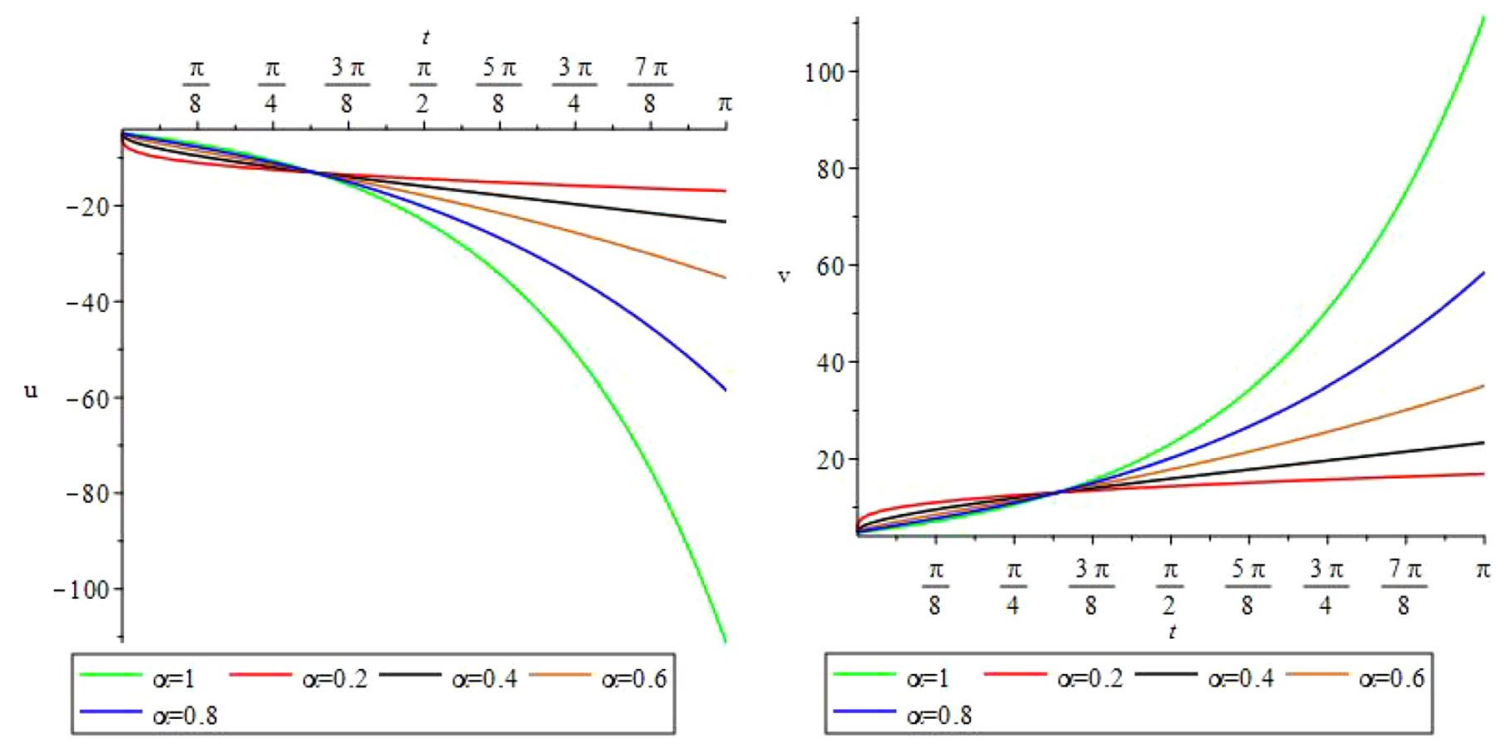

Fig. 11 Solution plots of Eq. (47) for different values of $\alpha$ (Example 2)

$$
\begin{aligned}
& E\{v(x, y, z, t)\}=q^{2}(x-0.5 y+z) \\
& \quad+q^{\alpha} E\left[\rho_{0}\left(\frac{\partial^{2} v}{\partial x^{2}}+\frac{\partial^{2} v}{\partial y^{2}}+\frac{\partial^{2} v}{\partial z^{2}}\right)-u \frac{\partial v}{\partial x}-v \frac{\partial v}{\partial y}-w \frac{\partial v}{\partial z}\right]
\end{aligned}
$$

$E\{w(x, y, z, t)\}=q^{2}(x+y-0.5 z)$

$$
+q^{\alpha} E\left[\rho_{0}\left(\frac{\partial^{2} w}{\partial x^{2}}+\frac{\partial^{2} w}{\partial y^{2}}+\frac{\partial^{2} w}{\partial z^{2}}\right)-u \frac{\partial w}{\partial x}-v \frac{\partial w}{\partial y}-w \frac{\partial w}{\partial z}\right]
$$

The inverse Elzaki transform of Eqs. (49)-(51) implies that

$$
\begin{aligned}
& u(x, y, z, t)=(-0.5 x+y+z) \\
& +E^{-1}\left[q^{\alpha} E\left[\rho_{0}\left(\frac{\partial^{2} u}{\partial x^{2}}+\frac{\partial^{2} u}{\partial y^{2}}+\frac{\partial^{2} u}{\partial z^{2}}\right)-u \frac{\partial u}{\partial x}-v \frac{\partial u}{\partial y}-w \frac{\partial u}{\partial z}\right]\right] \\
& v(x, y, z, t)=(x-0.5 y+z) \\
& +E^{-1}\left[q^{\alpha} E\left[\rho_{0}\left(\frac{\partial^{2} v}{\partial x^{2}}+\frac{\partial^{2} v}{\partial y^{2}}+\frac{\partial^{2} v}{\partial z^{2}}\right)-u \frac{\partial v}{\partial x}-v \frac{\partial v}{\partial y}-w \frac{\partial v}{\partial z}\right]\right]
\end{aligned}
$$


$w(x, y, z, t)=(x+y-0.5 z)$

$+E^{-1}\left[q^{\alpha} E\left[\rho_{0}\left(\frac{\partial^{2} w}{\partial x^{2}}+\frac{\partial^{2} w}{\partial y^{2}}+\frac{\partial^{2} w}{\partial z^{2}}\right)-u \frac{\partial w}{\partial x}-v \frac{\partial w}{\partial y}-w \frac{\partial w}{\partial z}\right]\right]$

Now applying the homotopy perturbation method we obtain

$$
\begin{aligned}
& \sum_{n=0}^{\infty} p^{n} u_{n}(x, y, z, t)=(-0.5 x+y+z) \\
& +p\left\{E^{-1}\left[q^{\alpha} E\left[\sum_{n=0}^{\infty} p^{n} H_{n}(u)\right]\right]\right\} \\
& \sum_{n=0}^{\infty} p^{n} v_{n}(x, y, z, t)=(x-0.5 y+z)+p\left\{E^{-1}\left[q^{\alpha} E\left[\sum_{n=0}^{\infty} p^{n} H_{n}(v)\right]\right]\right\}
\end{aligned}
$$

$$
\begin{aligned}
& \sum_{n=0}^{\infty} p^{n} w_{n}(x, y, z, t)=(x+y-0.5 z) \\
& +p\left\{E^{-1}\left[q^{\alpha} E\left[\sum_{n=0}^{\infty} p^{n} H_{n}(w)\right]\right]\right\}
\end{aligned}
$$

where $H_{n}(u), H_{n}(v)$ and $H_{n}(w)$ are He's polynomials that represents the nonlinear terms as

$$
\left\{\begin{array}{l}
H_{n}(u)=p\left[\rho_{0}\left(\frac{\partial^{2} u}{\partial x^{2}}+\frac{\partial^{2} u}{\partial y^{2}}+\frac{\partial^{2} u}{\partial z^{2}}\right)-u \frac{\partial u}{\partial x}-v \frac{\partial u}{\partial y}-w \frac{\partial u}{\partial z}\right]=0 \\
H_{n}(v)=p\left[\rho_{0}\left(\frac{\partial^{2} v}{\partial x^{2}}+\frac{\partial^{2} v}{\partial y^{2}}+\frac{\partial^{2} v}{\partial z^{2}}\right)-u \frac{\partial v}{\partial x}-v \frac{\partial v}{\partial y}-w \frac{\partial v}{\partial z}\right]=0 \\
H_{n}(w)=p\left[\rho_{0}\left(\frac{\partial^{2} w}{\partial x^{2}}+\frac{\partial^{2} w}{\partial y^{2}}+\frac{\partial^{2} w}{\partial z^{2}}\right)-u \frac{\partial w}{\partial x}-v \frac{\partial w}{\partial y}-w \frac{\partial w}{\partial z}\right]=0
\end{array}\right.
$$

where

$$
\begin{gathered}
u=u_{0}+p u_{1}+p^{2} u_{2}+\cdots, \\
v=v_{0}+p v_{1}+p^{2} v_{2}+\cdots, \\
w=w_{0}+p w_{1}+p^{2} w_{2}+\cdots,
\end{gathered}
$$

The first few components of He's polynomials are given as

$$
\left\{\begin{array}{l}
H_{0}(u)=\rho_{0}\left(u_{0 x x}+u_{0 y y}+u_{0 z z}\right)-u_{0} u_{0 x}-v_{0} u_{0 y}-w_{0} u_{0 z} \\
H_{0}(v)=\rho_{0}\left(v_{0 x x}+v_{0 y y}+v_{0 z z}\right)-u_{0} v_{0 x}-v_{0} v_{0 y}-w_{0} v_{0 z} \\
H_{0}(w)=\rho_{0}\left(w_{0 x x}+w_{0 y y}+w_{0 z z}\right)-u_{0} w_{0 x}-v_{0} w_{0 y}-w_{0} w_{0 z} \\
H_{1}(u)=\rho_{0}\left(u_{1 x x}+u_{1 y y}+u_{1 z z}\right)-u_{0} u_{1 x}-u_{1} u_{0 x}-v_{0} u_{1 y}-v_{1} u_{0 y}-w_{0} u_{1 z}-w_{1} u_{0 z} \\
H_{1}(v)=\rho_{0}\left(v_{1 x x}+v_{1 y y}+v_{1 z z}\right)-u_{0} v_{1 x}-u_{1} v_{0 x}-v_{0} v_{1 y}-v_{1} v_{0 y}-w_{0} v_{1 z}-w_{1} v_{0 z} \\
H_{1}(w)=\rho_{0}\left(w_{1 x x}+w_{1 y y}+w_{1 z z}\right)-u_{0} w_{1 x}-u_{1} w_{0 x}-v_{0} w_{1 y}-v_{1} w_{0 y}-w_{0} w_{1 z}-w_{1} w_{0 z} \\
\vdots
\end{array}\right.
$$

Using the above He's polynomials and comparing the coefficients of same power of $p$ in Eqs. (55)-(57) we have

$$
\begin{aligned}
p^{0}: u_{0}(x, y, z, t) & =(-0.5 x+y+z) \\
v_{0}(x, y, z, t) & =(x-0.5 y+z) \\
w_{0}(x, y, z, t) & =(x+y-0.5 z) \\
p^{1}: u_{1}(x, y, z, t) & =E^{-1}\left[q^{\alpha} E\left[H_{0}(u)\right]\right]=-(2.25 x) \frac{t^{\alpha}}{\Gamma(\alpha+1)} \\
v_{1}(x, y, z, t) & =E^{-1}\left[q^{\alpha} E\left[H_{0}(v)\right]\right]=-(2.25 y) \frac{t^{\alpha}}{\Gamma(\alpha+1)} \\
w_{1}(x, y, z, t) & =E^{-1}\left[q^{\alpha} E\left[H_{0}(w)\right]\right]=-(2.25 z) \frac{t^{\alpha}}{\Gamma(\alpha+1)}
\end{aligned}
$$

$$
\begin{aligned}
p^{2}: u_{2}(x, y, z, t) & =E^{-1}\left[q^{\alpha} E\left[H_{1}(u)\right]\right] \\
& =(-2.25 x+4.5 y+4.5 z) \frac{t^{2 \alpha}}{\Gamma(2 \alpha+1)} \\
v_{2}(x, y, z, t) & =E^{-1}\left[q^{\alpha} E\left[H_{1}(v)\right]\right] \\
& =(4.5 x-2.25 y+4.5 z) \frac{t^{2 \alpha}}{\Gamma(2 \alpha+1)} \\
w_{2}(x, y, z, t) & =E^{-1}\left[q^{\alpha} E\left[H_{1}(w)\right]\right] \\
& =(4.5 x+4.5 y-2.25 z) \frac{t^{2 \alpha}}{\Gamma(2 \alpha+1)}
\end{aligned}
$$

$p^{3}: u_{3}(x, y, z, t)=E^{-1}\left[q^{\alpha} E\left[H_{2}(u)\right]\right]$

$$
=-\left(20.25+5.0625 \frac{\Gamma(2 \alpha+1)}{(\Gamma(\alpha+1))^{2}}\right) \frac{x t^{3 \alpha}}{\Gamma(3 \alpha+1)}
$$$$
v_{3}(x, y, z, t)=E^{-1}\left[q^{\alpha} E\left[H_{2}(v)\right]\right]
$$$$
=-\left(20.25+5.0625 \frac{\Gamma(2 \alpha+1)}{(\Gamma(\alpha+1))^{2}}\right) \frac{y t^{3 \alpha}}{\Gamma(3 \alpha+1)}
$$

$w_{3}(x, y, z, t)=E^{-1}\left[q^{\alpha} E\left[H_{21}(w)\right]\right]$

$$
=-\left(20.25+5.0625 \frac{\Gamma(2 \alpha+1)}{(\Gamma(\alpha+1))^{2}}\right) \frac{z t^{3 \alpha}}{\Gamma(3 \alpha+1)}
$$


So the solutions $u(x, y, z, t), v(x, y, z, t)$ and $w(x, y, z, t)$ are given as
This solution is same as solution solved in [38]. The plots of Eq. (61) are depicted in Fig. 12 for $\alpha=1, \rho_{0}=0.5, t=0.1$

$$
\begin{aligned}
& u(x, y, z, t)=u_{0}(x, y, z, t)+u_{1}(x, y, z, t)+u_{2}(x, y, z, t)+u_{3}(x, y, z, t) \cdots=(-0.5 x+y+z)+-(2.25 x) \frac{t^{\alpha}}{\Gamma(\alpha+1)}+ \\
& (-2.25 x+4.5 y+4.5 z) \frac{t^{2 \alpha}}{\Gamma(2 \alpha+1)}+-\left(20.25+5.0625 \frac{\Gamma(2 \alpha+1)}{(\Gamma(\alpha+1))^{2}}\right) \frac{x t^{3 \alpha}}{\Gamma(3 \alpha+1)}+\cdots
\end{aligned}
$$

$$
\begin{aligned}
& v(x, y, z, t)=v_{0}(x, y, z, t)+v_{1}(x, y, z, t)+v_{2}(x, y, z, t)+v_{3}(x, y, z, t) \cdots=(x-0.5 y+z)+-(2.25 y) \frac{t^{\alpha}}{\Gamma(\alpha+1)}+ \\
& (4.5 x-2.25 y+4.5 z) \frac{t^{2 \alpha}}{\Gamma(2 \alpha+1)}+-\left(20.25+5.0625 \frac{\Gamma(2 \alpha+1)}{(\Gamma(\alpha+1))^{2}}\right) \frac{y t^{3 \alpha}}{\Gamma(3 \alpha+1)}+\cdots
\end{aligned}
$$

$w(x, y, z, t)=w_{0}(x, y, z, t)+w_{1}(x, y, z, t)+w_{2}(x, y, z, t)+w_{3}(x, y, z, t) \cdots=(x+y-0.5 z)+-(2.25 z) \frac{t^{\alpha}}{\Gamma(\alpha+1)}+$

$$
(4.5 x+4.5 y-2.25 z) \frac{t^{2 \alpha}}{\Gamma(2 \alpha+1)}+-\left(20.25+5.0625 \frac{\Gamma(2 \alpha+1)}{(\Gamma(\alpha+1))^{2}}\right) \frac{z t^{3 \alpha}}{\Gamma(3 \alpha+1)}+\cdots
$$

For $\alpha=1$ Eqs. (58)-(60) reduce to and the comparison plots of $U_{0}, U_{1}, U_{2}, U_{3}, V_{0}, V_{1}, V_{2}, V_{3}$

$u(x, y, z, t)=(-0.5 x+y+z)\left(1+2.25 t^{2}+2.25^{2} t^{4}+\cdots\right)-2.25 x t\left(1+2.25 t^{2}+\cdots\right)=\frac{-0.5 x+y+z-2.25 x t}{1-2.25 t^{2}}$

$v(x, y, z, t)=(x-0.5 y+z)\left(1+2.25 t^{2}+2.25^{2} t^{4}+\cdots\right)-2.25 y t\left(1+2.25 t^{2}+\cdots\right)=\frac{x-0.5 y+z-2.25 y t}{1-2.25 t^{2}}$

$w(x, y, z, t)=(x+y-0.5 z)\left(1+2.25 t^{2}+2.25^{2} t^{4}+\cdots\right)-2.25 z t\left(1+2.25 t^{2}+\cdots\right)=\frac{x+y-0.5 z-2.25 z t}{1-2.25 t^{2}}$

Fig. 12 Solution plots of Eq. (61) for $t=0.1$ and $\alpha=1$ (Example 3)
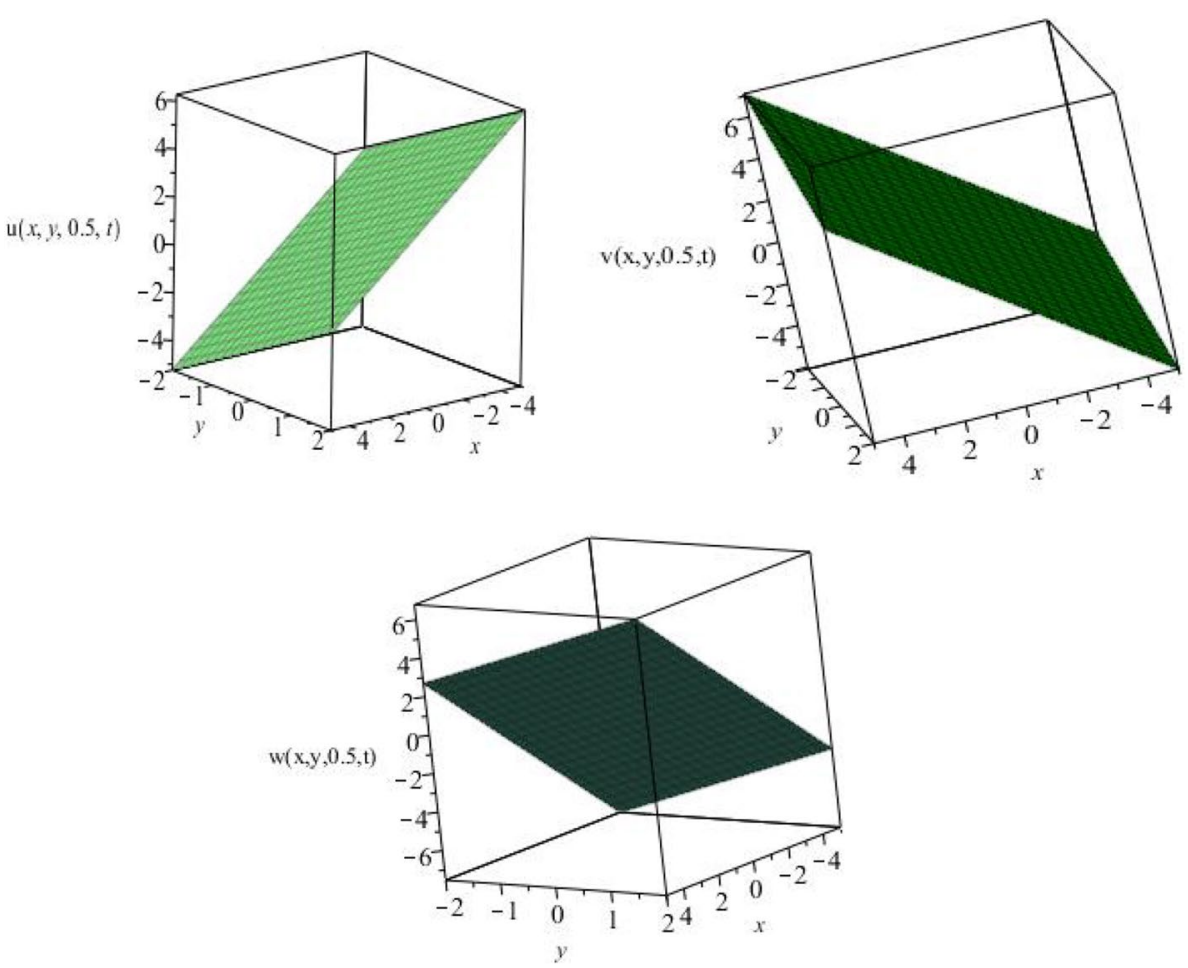

SN Applied Sciences 

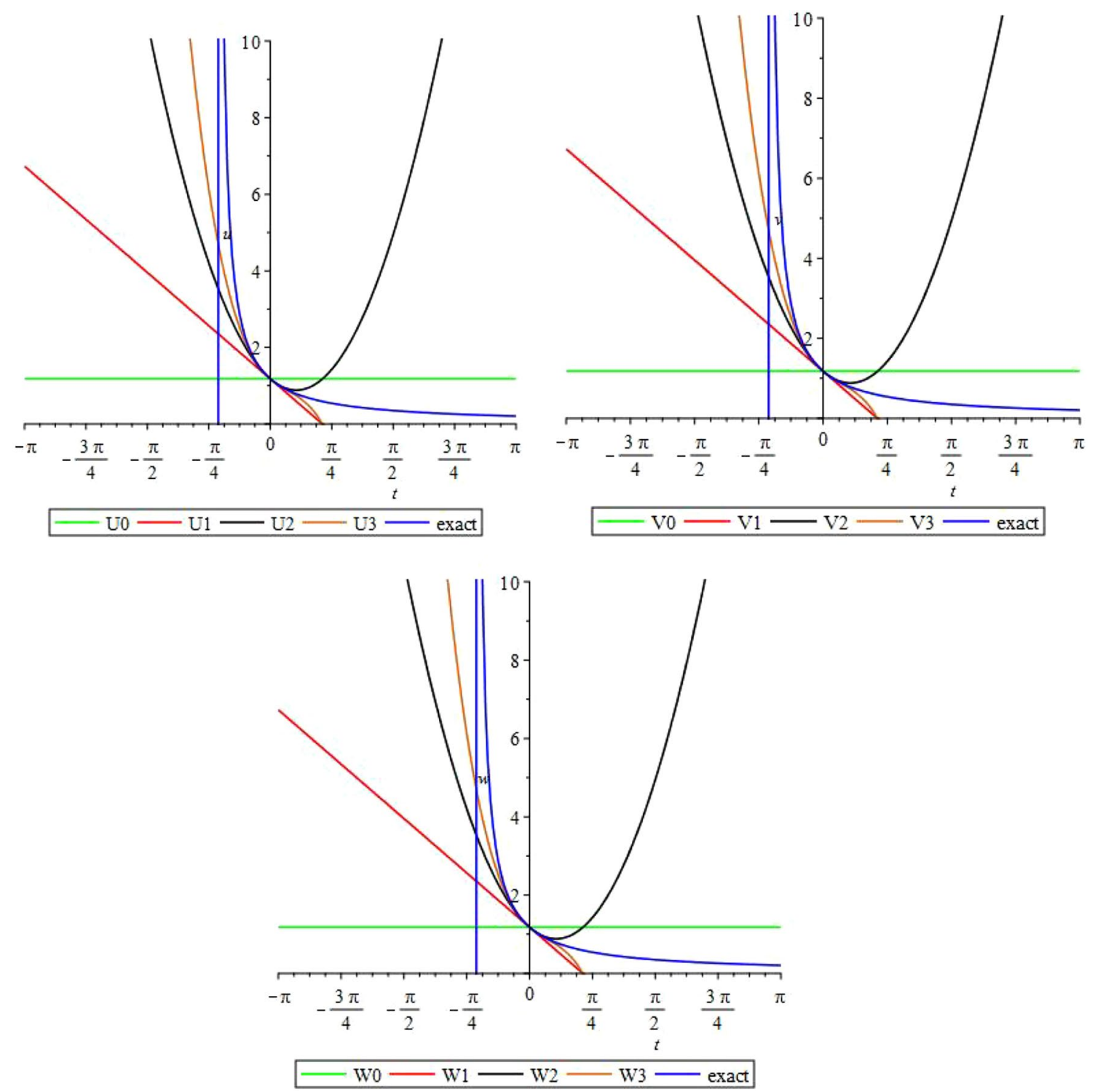

Fig. 13 Comparison of the approximate solution $U_{0}, U_{1}, U_{2}, U_{3}, V_{0}, V_{1}, V_{2}, V_{3}$ and $W_{0}, W_{1}, W_{2}, W_{3}$ with their exact solution (61) for $\rho_{0}=0.5, x=\frac{\pi}{4}, y=\frac{\pi}{4}, z=\frac{\pi}{4}$ (Example 3$)$

and $W_{0}, W_{1}, W_{2}, W_{3}$ with their exact solution (61) for $\alpha=1$ are depicted in Fig. 13.

\section{Conclusion}

In this paper, HPETM is applied for solution of time-fractional NS equations with IC. HPETM provides the solution in term of convergent series. Three example problems are addressed in order to validate and test the efficacy of the proposed method. One may see that the obtained results are in excellent agreement with HPM [28] and ADM [29]. The major benefit of this method over HPM and ADM is that this is a powerful and effective method in finding the analytical and approximate solutions for fractional order nonlinear partial differential equations in place of Adomian's polynomials.

Acknowledgements The first author expresses his sincere thanks to Department of Science and Technology, Govt. of India for providing INSPIRE fellowship (IF170207) to undertake the present work.

\section{Compliance with ethical standards}

Conflict of interest All authors declare that they have no conflict of interest.

Ethical approval This article does not contain any studies with human participants or animals performed by any of the authors. 


\section{References}

1. Kilbas AA, Srivastava HM, Trujillo JJ (2006) Theory and applications of fractional differential equations, vol 204. Elsevier, Amsterdam, $540 \mathrm{pp}$

2. Kiryakova V (1994) Generalized fractional calculus and applications. Pitman research notes in mathematics series, vol 301. Longman Scientific \& Technical, Harlow

3. Lakshmikantham V, Vatsala AS (2008) Basic theory of fractional differential equations. Nonlinear Anal 69:2677

4. Miller KS, Ross B (1993) An introduction to the fractional calculus and differential equations. Wiley, New York

5. Podlubny I (1999) Fractional differential equation. Academic Press, San Diego

6. Momani S, Odibat Z, Erturk VS (2007) Generalized differential transform method for solving a space-and time-fractional diffusion-wave equation. Phys Lett A 370:379

7. Odibat Z, Momani S (2008) A generalized differential transform method for linear partial differential equations of fractional order. Appl Math Lett 21:194

8. Zhang Y (2009) A finite difference method for fractional partial differential equation. Appl Math Comput 215:524

9. Wang Q (2006) Numerical solutions for fractional KdV-Burgers equation by Adomian decomposition method. Appl Math Comput 182:1048

10. Daftardar-Gejji V, Bhalekar S (2008) Solving multi-term linear and non-linear diffusion-wave equations of fractional order by Adomian decomposition method. Appl Math Comput 202:113

11. Wang Q (2007) Homotopy perturbation method for fractional KdV equation. Appl Math Comput 190:1795

12. Wang Q (2008) Homotopy perturbation method for fractional KdV-Burgers equation. Chaos Solitons Fractals 35:843

13. Abdulaziz O, Hashim I, Ismail ES (2009) Approximate analytical solution to fractional modified KdV equations. Math Comput Model 49:136

14. Rahman MU, Khan RA (2013) Numerical solutions to initial and boundary value problems for linear fractional partial differential equations. Appl Math Model 37:5233

15. Akinlar MA, Secer A, Bayram M (2014) Numerical solution of fractional Benney equation. Appl Math Inf Sci 8:1633

16. Secer A, Akinlar MA, Cevikel A (2012) Similarity solutions for multiterm time-fractional diffusion equation. Adv Differ Equ 2012:7

17. Kurulay M, Bayram M (2010) Approximate analytical solution for the fractional modified KdV by differential transform method. Commun Nonlinear Sci Numer Simulat 15:17

18. Kurulay M, Akinlar MA, Ibragimov R (2013) Computational solution of a fractional integro-differential equation. Abstr Appl Anal 2013:4

19. Chamekh M, Elzaki TM (2018) Explicit solution for some generalized fluids in laminar flow with slip boundary conditions. J Math Computer Sci 18:272

20. Elzaki TM, Chamekh M (2018) Solving nonlinear fractional differential equations using a new decomposition method. Univ J Appl Math Comput 6:27

21. Alderremy AA, Elzaki TM, Chamekh M (2018) New transform iterative method for solving some Klein-Gordon equations. Results Phys 10:655
22. Gad-Allah MR, Elzaki TM (2018) Application of new homotopy perturbation method for solving partial differential equations. J Comput Theor Nanosci 15:500

23. Gad-Allah MR, Elzaki TM (2017) Application of the new homotopy perturbation method (NHPM) for solving non-linear partial differential equations. J Comput Theor Nanosci 14:1

24. Navier CLMH (1822) Mémoire sur les lois du mouvement des fluides. Mem Acad Sci Inst France 6:389

25. El-Shahed M, Salem A (2005) On the generalized Navier-Stokes equations. Appl Math Comput 156:287

26. Kumar D, Singh J, Kumar S (2015) A fractional model of NavierStokes equation arising in unsteady flow of a viscous fluid. J Assoc Arab Univ Basic Appl Sci 17:14

27. Ragab AA, Hemida KM, Mohamed MS, Abd El Salam MA (2012) Solution of time-fractional Navier-Stokes equation by using homotopy analysis method. Gen Math Notes 13:13

28. Ganji ZZ, Ganji DD, Ganji AD, Rostamian M (2010) Analytical solution of time-fractional Navier-Stokes equation in polar coordinate by homotopy perturbation method. Numer Methods Part Diff Equ 26:117

29. Birajdar GA (2014) Numerical solution of time fractional NavierStokes equation by discrete Adomian decomposition method. Nonlinear Eng. 3:21

30. Momani S, Odibat Z (2006) Analytical solution of a time-fractional Navier-Stokes equation by Adomian decomposition method. Appl Math Comput 177:488

31. Kumar S, Kumar D, Abbasbandy S, Rashidi MM (2014) Analytical solution of fractional Navier-Stokes equation by using modified Laplace decomposition method. Ain Shams Eng J 5:569

32. Chaurasia VBL, Kumar D (2011) Solution of the time-fractional Navier-Stokes equation. Gen Math Notes 4:49

33. Caputo M, Mainardi F (1971) Linear models of dissipation in anelastic solids. Rivist Nuovo Cimento 1:161

34. Carpinteri A, Mainardi F (1997) Wien. Springer, New York

35. Elzakim TM, Elzaki SM, Hilal EMA (2012) Elzaki and Sumudu transforms for solving some differential equations. Glob J Pure Appl Math 8:167

36. Elzaki TM, Elzaki SM (2011) On the connections between Laplace and Elzaki transforms. Adv Theor Appl Math 6:1

37. Singh BK, Kumar P (2016) FRDTM for numerical simulation of multi-dimensional, time-fractional model of NavierStokes equation. Ain Shams Eng J. https://doi.org/10.1016/j. asej.2016.04.009

38. Campos MD, Romao EC (2014) A high-order finite-difference scheme with a linearization technique for solving of threedimensional Burgers equation. Comput Model Eng Sci 103:139

39. Morales-Delgado VF, Gomez-Aguilar JF, Kumar S, TanecoHernandez MA (2018) Analytical solutions of the Keller-Segel chemotaxis model involving fractional operators without singular kernel. Eur Phys J Plus 133:200

40. Ghosh U, Banerjee J, Sarkar S, Das S (2018) Fractional Klein-Gordon equation composed of Jumarie fractional derivative and its interpretation by a smoothness parameter. Pramana J Phys 90:74 